\title{
Fourier Domain Mode Locking Laser Based On Two-Pump Optical Parametric Amplification
}

\author{
Kim K. Y. Cheung, Chi Zhang, Kyle H. Y. Cheng, M. H. Yeung,Yue Zhou and Kenneth K. Y. Wong* \\ Photonic Systems Research Laboratory, Department of Electrical and Electronic Engineering, \\ The University of Hong Kong, Pokfulam Road, Hong Kong. \\ * Presently on leave at the Massachusetts Institute of Technology, Cambridge, MA, USA. Email: kywong@eee.hku.hk
}

\begin{abstract}
We present a Fourier domain mode locked (FDML) laser scanning from 1540 to 1570 using two-pump optical parametric amplifier (OPA) as the gain medium. The sweep rate of $39.683 \mathrm{kHz}$ is achieved.
\end{abstract}

\section{INTRODUCTION}

Swept sources have been playing an important role in a series of biomedical imaging, spectroscopy and sensing applications [1-3]. Fourier domain mode locking technique is widely used to generate the swept sources due to the ease of implementation. In FDML, a laser cavity is constructed by a gain medium and a tunable optical bandpass filter which is driven by a periodical sinusoidal signal. It matches the fiber laser ring cavity with the round trip time of the photons circulating in the cavity such that when one wavelength arrives at the filter, it combines with the same particular wavelength that is being generated [4]. Various gain medium such as semiconductor optical amplifier (SOA) $[1,5]$. Raman amplifier (RA) [6] and erbium doped fiber amplifier (EDFA) [7] have been demonstrated in FDML. However for SOA and EDFA, their amplification windows are limited by the material properties, the sweeping wavelength of FDML is fixed. While for RA, it was shown that the sweeping range was about $30 \mathrm{~nm}$ [6]. Thanks for the femtosecond response time [8], high gain [9] and wide gain bandwidth [10] provide by OPA, its gain regime can be varied by changing the position of the pump. Hybrid FDML using OPA has been demonstrated by Cheng et al. [11] using polygon filter as the tunable filter and with an extra EDFA adding inside the cavity to complement the dip due to gain spectrum of one-pump OPA. However the central dip could not be completely be compensated by the EDFA hence a disjoint in the sweeping spectrum was inevitable. A central spike between the up/down scanned pulses was observed which was due to the residue of the pump. In this paper, we propose and demonstrate a two-pump OPA FDML which can sweep continuously without a disjoint in frequency spectrum and without a central spike between up/down scan pulses as the pumps can be completely removed.

\section{EXPERIMENTAL SETUP}

The schematic diagram for the FDML wavelength swept laser based on two-pump OPA is shown in Fig. 1. Two pumps were generated by a continuous-wave (CW) tunable laser source (TLS1 and TLS2) with wavelengths at $1535 \mathrm{~nm}$ and $1585 \mathrm{~nm}$ respectively. They were then phased modulated by phase modulators (PM) with $1 \mathrm{Gbps} 2^{23}-1$ pseudo-random bit sequence (PRBS) in order to suppress the stimulated Brillouin scattering (SBS) effect [12].

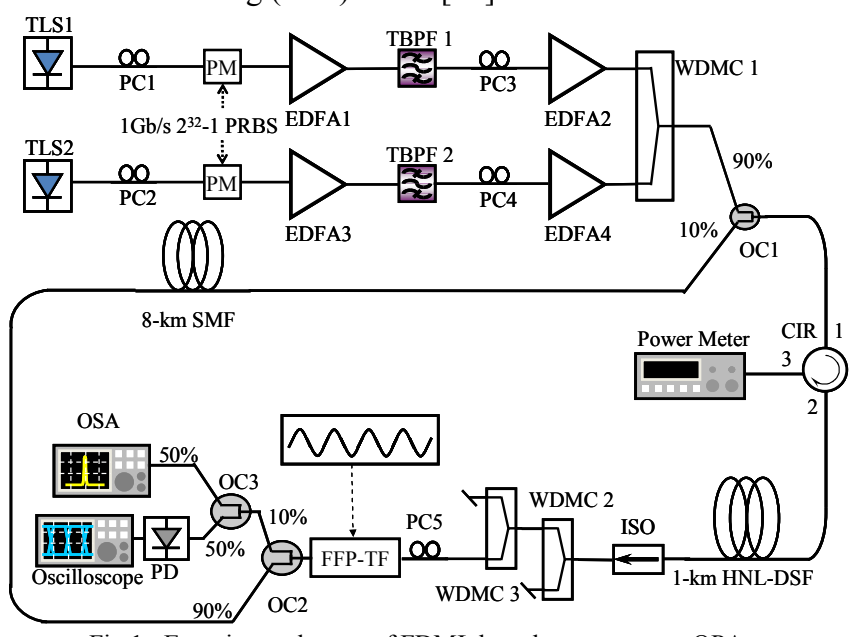

Fig.1. Experimental setup of FDML based on two-pump OPA.

The polarization controllers (PC1 and $\mathrm{PC} 2$ ) were used to align the state of polarization (SOP) of the pumps to the PMs. The dithering pumps were then amplified by twostage EDFAs, C-band EDFA (EDFA1 and EDFA2) and Lband EDFA (EDFA 3 and EDFA4), with tunable bandpass filters (TBPF) inserted between the two EDFAs to suppress amplified spontaneous emission (ASE) noise. They were combined together by wavelength division multiplexing coupler (WDMC1) before launching into a 1-km highly nonlinear dispersion-shifted fiber (HNL-DSF) with zerodispersion wavelength (ZDW) at $1560 \mathrm{~nm}$, dispersion slope of $0.024 \mathrm{ps} / \mathrm{nm}^{2} / \mathrm{km}$ and nonlinear coefficient $\gamma=14 \mathrm{~W}^{-1} \mathrm{~km}^{-1}$. A circulator (CIR) was used to prevent the reflected pump power. The input C-band pump was $26 \mathrm{dBm}$ while the input L-band pump was $24 \mathrm{dBm}$. To prevent pumps oscillating inside the cavity and cause damage, WDMC 2 and WDMC 3 were used to remove the residue pumps. In order to enable FDML operation, the Fabry-Perot filter (FFP-TF) was driven by a sinusoidal wave periodically with a period matched to the optical round-trip time of the laser cavity, or a harmonic thereof. The FFP-TF used has a free spectral range (FSR) of $\sim 160 \mathrm{~nm}$ at $1550 \mathrm{~nm}$ and a finesse of $\sim 750$. To reduce the driving frequency required to synchronize the cavity round trip time, an 8-km single-mode fiber (SMF) was added inside the cavity. Isolator (ISO) in the cavity is to enable uni- 
directional operation. A 10/90 optical coupler (OC2) in the cavity provided $90 \%$ feedback signal and $10 \%$ output. $90 \%$ feedback signal was then combined with the pump by OC1 while $10 \%$ output signal was monitored by an optical spectrum analyzer (OSA) and oscilloscope.

\section{EXPERIMENTAL RESULTS AND DISCUSSIONS}

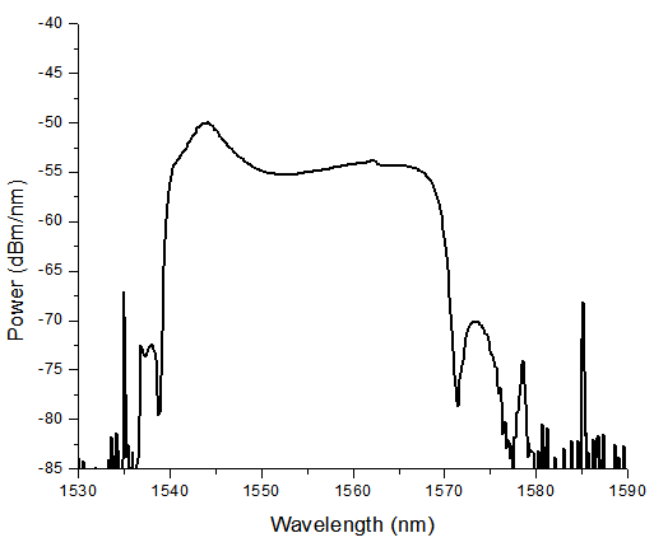

Fig.2. FDML spectrum based on two-pump OPA.

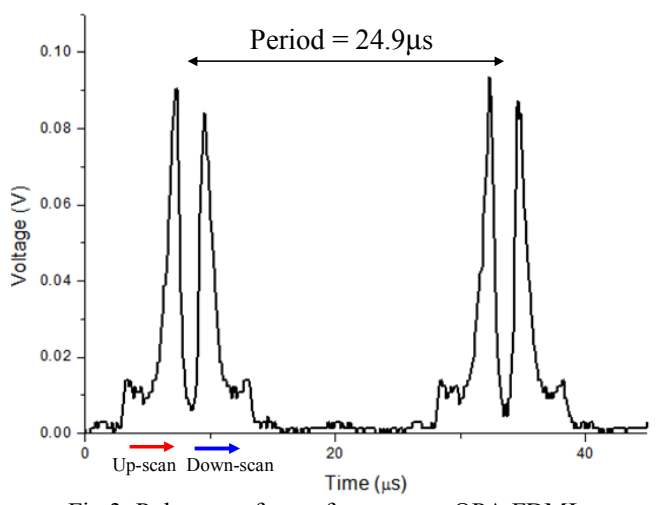

Fig.3. Pulse waveform of two-pump OPA FDML.

As shown in Fig. 2, two-pump OPA FDML generates a continuous spectrum as expected. The two spikes locate at $1535 \mathrm{~nm}$ and $1585 \mathrm{~nm}$ are the residual pumps. The sweeping range is from $1540 \mathrm{~nm}$ to $1570 \mathrm{~nm}$. The sweeping range of FDML depends on the two-pump OPA gain spectrum and broader the spectrum is desired. Broader OPA gain spectrum can be obtained by increasing the pump power and changing the wavelengths of two pumps. In our case, the sweeping range is restricted by the transmission port of the WDMC 2 and WDMC 3 which were used to filter the pump away. It is believed that the problem can be solved by using two FBGs to filter the two pumps instead of using WDMC.

Fig. 3 shows the pulse waveform of the two-pump OPA FDML. We defined up-scan as the case where filter is tuning from short to long wavelength while the opposite occurs in down-scan. It can be observed clearly pulse waveform of the two-pump OPA FDML shows a more coherent pulse and there is no notch on either up-scan or down-scan pulse when comparing with [11]. The period between the pulses is $24.9 \mu$ s which matches well with the driving frequency of the FFP-TF. The output power is -13.5 $\mathrm{dBm}$. The low output power is due to $\mathrm{OC} 1$ where only $10 \%$ of $90 \%$ of feedback are combined with the pump. The output power could be increased by replacing $\mathrm{OC} 1$ with a proper WDMC.

\section{CONCLUSION}

In this paper, we proposed and demonstrated an FDML wavelength-swept fiber laser using two-pump OPA as the gain medium. The sweeping range was from $1540 \mathrm{~nm}$ to $1570 \mathrm{~nm}$ with output power $-13.5 \mathrm{dBm}$ at sweep rate of $39.7 \mathrm{KHz}$. The output spectrum and pulse waveforms are observed. The pulses obtained can resemble a Gaussian shape which is suitable for Optical coherence tomography.

\section{ACKNOWLEDGMENT}

The work described in this paper was partially supported by grants from the Research Grants Council of the Hong Kong Special Administrative Region, China (Project No. HKU 7179/08E and HKU 7183/09E). The authors would also like to acknowledge Sumitomo Electric Industries for providing the HNL-DSF.

\section{REFERENCE}

[1] R. Huber, M. Wojtkowski, and J. G. Fujimoto, "Fourier Domain Mode Locking (FDML): A new laser operation regime and applications for opical coherence tomography," Opt. Express 14(8), 3225-3237 (2006).

[2] E. J.Jung, C. S. Kim, M. Y. Jeong, M. K. Kim, M. Y. Jeon, W.Jung, and Z. P. Chen, "Characterization of FBG sensor interrogation based on FDML wavelength swept laser," Opt. Express 16(21), 16552-16560 (2008).

[3] S. W. Huang, A. D. Aguirre, R. A Huber, D. C. Adler, and J. G. Fujimoto, "Swept source optical coherence microscopy using a Fourier domain mode-locked laser," Opt. Express 15(10), 6210-6217 (2007).

[4] R. Leonhardt, B. R. Bidermann, W. Wieser, and R. Huber, "Nonlinear optical frequency conversion of an amplified Fourier Domain Mode Locked (FDML) laser," Opt. Express 17(19), 16801-16808 (2009).

[5] T. Kreatschmer, and S. T. Sanders, "Ultrastable Fourier Domain Mode Locking observed in a laser sweeping 1363.8-1363.7nm," in Conference on Lasers and Electro-Optics/International Quantum Electronics Conference, OSA Technical Digest (CD), paper CFB4, (2009).

[6] T. Klein, W. Wieser, B. R. Bidermann, C. M. Eigenwilling, G. Palte, and R. Huber, "Raman-pumped Fourier-domain mode-locked kaser: analysis of operation and application for optical coherence tomography," Opt. Letters 33(23), 2815-2817 (2008).

[7] S. L. Hyung, J. J. Eun, N. S. Seung, Y. J. Myung, and S. K. Chang, "FDML wavelength-swept fiber laser based on EDF gain medium" in IEEE 14th OptoElectronics and Communications Conference (OECC), paper FA5, (2009).

[8] M. E. Marhic, "Fiber Optical Parametric Amplifiers, Oscillators and related devices," Cambridge, UK: Canbridge Univ. Press, 2007.

[9] T. Torounidis, P. A. Andrekson, and B. E. Olsson, "Fiber-optical parametric amplifier with 70-dB gain," IEEE Photon. Technol. Lett., 18(10), 1194-1196 (2006).

[10] M. E. Marhic, K. K. Y. Wong, and L. G. Kazovsky, "Wide-band tuning of the gain spectrum of one pump fiber optical parametric amplifiers," IEEE J. Select. Topics Quantum Electron., 15(5), 1133-1141 (2004).

[11] K. H. Y. Cheng, Beau A. Standish, Victor X. D. Yang, K. K. Y. Cheung, Xijia Gu, Edmund Y. Lam, and K. K. Y. Wong, "Wavelength-swept spectral and pulse shaping utilizing hybrid Fourier domain modelocking by fiber optical parametric and erbium-doped fiber amplifiers," Opt. Express 18, 1909-1915 (2010)

[12] S. K. Korotky, P. B. Hansen, L. Eskildsen, and J. J. Veselka, "Efficient phase modulation scheme for suppressing stimulated Brillouin scattering," in Tech. Dig. Int. Conf. Integrated Optics and Optical Fiber Communications 2, paper WD2-1, (1995). 